\title{
Im Fokus: Das Praxissemester zukünftiger Russischlehrkräfte Einstellungen und Einschätzungen Berliner Lehramtsstudierender
}

Taking into account the necessity of closer interrelation between the university education and school practice, the first part of the paper investigates what benefits future teachers of Russian as a foreign language can gain before and during the practical semester from the new type of advising offered by the lecturer in the second phase of education as well as from the support of the specially trained in-service teachers. The second part focuses on the results of a survey, conducted with seven students at the beginning and end of the practical semester 2017/18. The survey revealed students' attitudes to and opinions on the preparation and the practical semester, among other things on how they evaluate the interrelation between theory and practice, as well as on the role of individual courses and different forms of support offers.

Исходя из необходимости более тесной взаимосвязи между университетским образованием и школьной практикой, в первой части статьи рассматривается вопрос, какую пользу могут извлечь будущие преподаватели русского языка до и во время прохождения семестровой школьной практики из нововведённой формы консультации, предлагаемой руководителем семинара во второй фазе обучения, а также из кураторства специально подготовленных учителей-практиков. В центре внимания второй части статьи находятся результаты опроса, проведённого в начале и в конце семестровой практики 2017/18 гг. среди семи студентовпрактикантов. При опросе были выявлены представления и мнения студентов по поводу подготовки и прохождения школьной практики, 
в том числе - как они оценивают взаимосвязь между теорией и практикой, а также вклад отдельных семинаров и различных форм сопровождения практики.

\section{Das Praxissemester für Lehramtsstudierende in Berlin}

Seit dem Wintersemester 2016/17 absolvieren alle Lehramtsstudierenden der Berliner Universitäten im 3. Semester ihres Masterstudiums ein fünfmonatiges Praxissemester an einer Berliner Schule. Das Praxissemester ist an die Stelle der bisherigen vierwöchigen Unterrichtspraktika in beiden Fächern getreten und folgt somit der bereits 2005 von der Kultusministerkonferenz (KMK) ausgesprochenen Empfehlung, den „Anteil der schulpraktischen Studien deutlich zu erhöhen und diese sowie die Bildungs- und Fachwissenschaften und deren Didaktiken [...] stärker miteinander zu vernetzen, um eine verbesserte Orientierung an den Erfordernissen des Lehrerberufes zu erreichen“ (KMK 2005: 2). Dementsprechend heißt es im „Leitfaden Praxissemester“ (2018: 7): „Ziel des Praxissemesters ist eine stärkere Verknüpfung von Universität und Schulpraxis." Diesem Anliegen und somit der besseren Verzahnung der drei Phasen der Lehrkräftebildung sehen sich all diejenigen verpflichtet, die in die Ausbildung und Lernbegleitung der Lehramtsstudierenden im Praxissemester involviert sind und die Professionalisierung der Studierenden in diesem Ausbildungsabschnitt unterstützen. 


\subsection{Die LernbegleiterInnen im Praxissemester}

In der Vorbereitung, Durchführung und Auswertung ihres Praxissemesters werden die Studierenden bzw. PraktikantInnen durch Lehrkräfte der Fachdidaktiken und Erziehungswissenschaften sowie MentorInnen und FachberaterInnen angeleitet und begleitet. Diese LernbegleiterInnen agieren in Abhängigkeit vom (Lehr-)Veranstaltungstyp und Lernort (vgl. Abbildung 1), die in den speziellen Modulen der Studien- und Prüfungsordnungen der jeweiligen Masterstudiengänge festgelegt sind. So absolvieren die Studierenden im Rahmen des „Moduls 5: Planung, Durchführung und Reflexion von Russischunterricht (Schulpraktikum)“ im Sommersemester ein Vorbereitungsseminar, vom 1. September bis 31. Januar das Schulpraktikum und parallel dazu bzw. im Anschluss ein Begleit- oder Nachbereitungsseminar (vgl. Fachspezifische StO/PO 2015: 13-14).

Für das Fach Russisch liegen das Vorbereitungsseminar und das Begleit/Nachbereitungsseminar in der Verantwortung der Fachdidaktikerin, die im konkreten Fall von einem Fachberater unterstützt wird. Während des Schulpraktikums sind die MentorInnen auf Grund ihrer räumlichen und zeitlichen Präsenz die wichtigsten Bezugspersonen für die PraktikantInnen. Unterstützung erfahren die Studierenden darüber hinaus auch durch die Fachdidaktikerin und den Fachberater. 


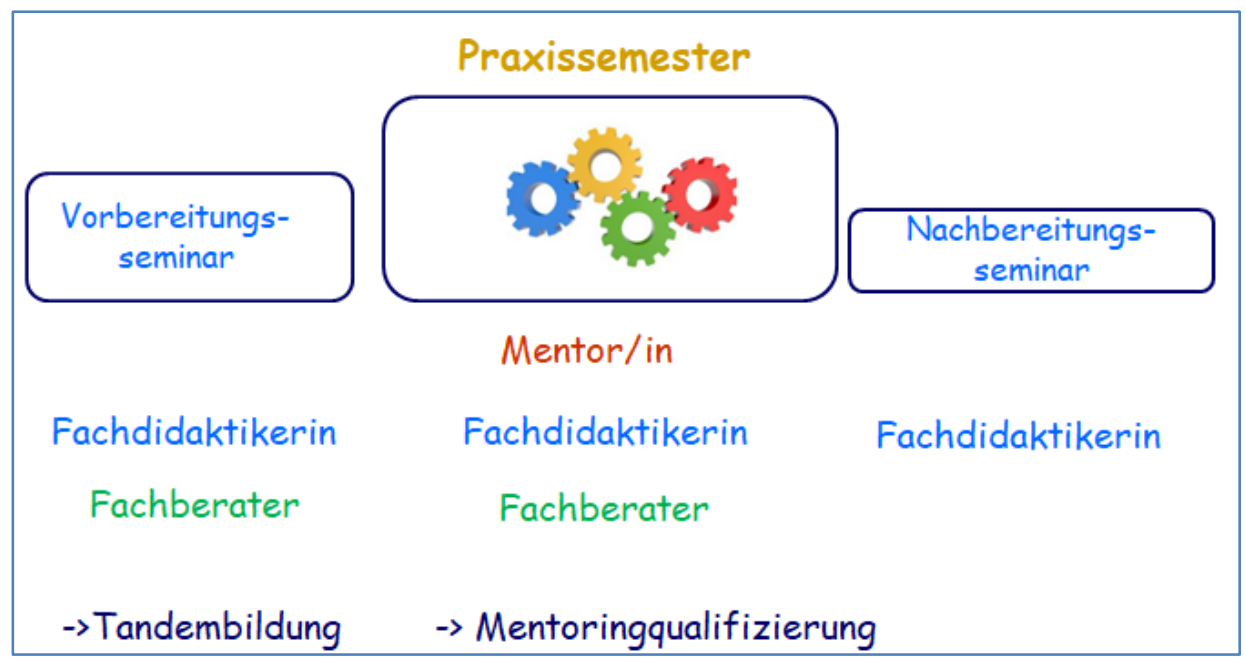

Abb. 1 LernbegleiterInnen in den verschiedenen Veranstaltungsformen während des Praxissemesters

Für eine phasenübergreifende Kooperation ist es sinnvoll, wenn die verschiedenen LernbegleiterInnen nicht nur ihr Selbstverständnis offenlegen (vgl. Koch 2015: 87), sondern sich zu ihren Rollen und Aufgaben austauschen und die konkreten Arbeitsfelder untereinander abstimmen. Dazu können und sollen universitätsübergreifende Angebote, wie die Fachberatung (siehe 1.2.) und die Mentoringqualifizierung (siehe 1.3.), die in Berlin bereits im Wintersemester 2016/17 gestartet sind, ihren Beitrag leisten. Bringen sie doch nicht nur die FachdidaktikerInnen, FachseminarleiterInnen (FachberaterInnen) und die LehrerInnen (MentorInnen) zusammen, sondern initiieren und unterstützen aktiv deren Austausch und ihre direkte Zusammenarbeit. Im Fachbereich Russisch kamen diese Angebote im ersten Durchlauf noch nicht zum Einsatz, sollten aber im folgenden Jahr Berücksichtigung finden. 


\subsection{Fachberatung}

Die Fachberatung gilt als innovatives Element im Praxissemester (vgl. Leitfaden 2018: 18), das auf der Grundlage einer engen Kooperation von Fachseminarleitung und Fachdidaktik eine Möglichkeit der Verzahnung von 1. und 2. Phase der Ausbildung aufzeigt.

Um die Fachberatung im Wintersemester 2017/18 auch für das Fach Russisch anbieten zu können, wurde bereits im Dezember 2016 ein Tandem aus Fachdidaktikerin und Fachseminarleiter, der im Praxissemester als Fachberater auftritt, gebildet. Im 1. Halbjahr 2017 durchlief dieses Tandem erfolgreich die entsprechenden Qualifizierungsmaßnahmen und plante dann entsprechend den Vorgaben die Integration der Fachberatung in das Vorbereitungsseminar und das Praxissemester. Im Ergebnis gestaltete der Fachberater gemeinsam mit der Fachdidaktikerin im Sommersemester 2017 eine Sitzung zur Strukturierung und Phasierung einer Unterrichtsstunde und eine weitere zur Differenzierung. Die Studierenden lernten so bereits vor dem Praxissemester ihren Fachberater kennen, der sie dann im Oktober in das Verfahren des „Kollegialen Unterrichtscoachings“ nach Kreis \& Staub (2013) einführte und ihnen exemplarisch ko-konstruktive Beratungsgespräche (als Beispiel wurde u.a. das selbst aufgezeichnete Beratungsgespräch zwischen Fachberater und Fachdidaktikerin gezeigt) sowie konkrete Beratungstechniken vorstellte, die dann von den PraktikantInnen in eigenen Beratungsgesprächen angewendet, aufgezeichnet und mit dem Fachberatertandem reflektiert wurden. Darüber hinaus ermöglichte der Fachberater den PraktikantInnen nicht nur den Besuch in seinem Unterricht, an den sich eine reflektierte Auswertung anschloss, sondern auch die Teilnahme an einer Fachseminarsitzung, damit sie bereits vor Antritt des Vorbereitungsdienstes (Referendariats) einen Einblick in die Themen und Arbeitsweisen eines Fachseminars erhalten. 


\subsection{Mentoringqualifizierung}

Mit der Überzeugung, dass qualifizierte MentorInnen einen wichtigen Gelingensfaktor im Praxissemester darstellen, wurde im Juni 2017 im Rahmen der Mentoringqualifizierung (MQ) erstmalig das Modul „Fachdidaktik Russisch" im Tandem von Fachdidaktikerin und einer Fachseminarleiterin angeboten. Damit hatten nun auch RussischlehrerInnen die Möglichkeit, eine MQ abzuschließen und entsprechend vorbereitet die Mentorentätigkeit zu übernehmen. Im Rahmen der insg. 19,5 Stunden umfassenden MQ absolvierten die RussischlehrerInnen zwei Module zur Lernbegleitung (7,5 Stunden) mit Schwerpunkt auf den Orientierungsgesprächen und den ko-konstruktiven Unterrichtsbesprechungen sowie ein Modul Fachdidaktik (12 Stunden). Letzteres verteilte sich auf vier Blöcke à drei Zeitstunden mit folgenden Themenstellungen:

1. Voraussetzungen für das Praxissemester und Anforderungen an die PraktikantInnen, Aufgaben der MentorInnen

2. Russischunterricht planen: Von der Unterrichtsreihe zur einzelnen Unterrichtsstunde, Differenzierung im Russischunterricht

3. Fachdidaktische Fragestellungen: Kompetenzorientierung, Methoden, Medien im Russischunterricht, Sprachbildung

4. Einführung in fachspezifische ko-konstruktive Beratungsgespräche mit Fallbeispiel, Gestaltung eines ko-konstruktiven Beratungsgespräches mit zukünftigen PraktikantInnen und deren Stundenentwürfen.

Die TeilnehmerInnen der MQ erwarben demzufolge nicht nur fachspezifische Kompetenzen für die zukünftige Lernbegleitung, sondern erhielten detaillierte Informationen sowohl zu den Abläufen und Studieninhalten im Masterstudium als auch zum Stand der Kompetenzentwicklung ihrer zukünftigen PraktikantInnen. 
Da im Vorfeld der MQ von der Fachdidaktikerin gezielt RussischlehrerInnen eingeladen wurden, die an potentiellen Praktikumsschulen für Russisch tätig sind, standen im Ergebnis acht MentorInnen für eine qualifizierte Betreuung im Praxissemester zur Verfügung. In der Vorbereitung des Praxissemesters ist es allerdings schwierig, eine Betreuung durch RussischlehrerInnen mit MQ zu garantieren, weil die Zuweisung der Studierenden auf die Schulen immer auch vom 2. Fach abhängt. Aus diesem Grund gilt es in Zukunft weitere RussischlehrerInnen für eine MQ zu gewinnen und somit die Anzahl der qualifizierten MentorInnen zu erhöhen.

\subsection{Vorbereitungsseminar}

Das Vorbereitungsseminar im Sommersemester 2017 umfasste insgesamt dreizehn Sitzungen mit dem Schwerpunkt „Planung von Unterricht“. Zwei Sitzungen wurden - wie bereits ausgeführt - im Tandem von Fachdidaktikerin und Fachberater zu den Themen „Strukturierung und Phasierung einer Unterrichtsstunde“ und zur „Differenzierung“ durchgeführt.

Die Studierenden durchliefen im Verlauf des Semesters die verschiedenen Elemente bzw. Aspekte der Unterrichtsplanung vom Allgemeinen zum Besonderen, d. h. von der Planung einer Reihe mit verschiedenen Kompetenzschwerpunkten bis zur Planung einer Einzelstunde mit ihren Phasen und entwickelten exemplarisch eine Reihe zum Thema „Meine Hobbies“. Für eine selbstgewählte Einzelstunde erarbeiteten sie einen Stundenentwurf (Verlaufsplan) und stellten ihn in der gemeinsamen Sitzung mit den TeilnehmerInnen der Mentoringqualifizierung in einem ko-konstruktiven Beratungsgespräch einer Russischlehrkraft vor. Bereits im Vorbereitungsseminar gelang es auf diese Art und Weise, den Studierenden die Verzahnung der Ausbildungsphasen aufzuzeigen und erlebbar zu machen. Die guten persönlichen Kontakte zwischen den Tandems aus Fachdidaktikerin 
und FachseminarleiterInnen und zu den einzelnen TeilnehmerInnen der Mentoringqualifizierung erleichterten den gegenseitigen Informationsaustausch und das abgestimmte Auftreten im Praxissemester.

\subsection{Aufgaben und Anforderungen im Praxissemester}

Die Lehramtsstudienordnungen fordern eine Präsenzzeit an der Schule mit 115 Stunden pro Fach (vgl. Fachspezifische StO/PO 2015: 14) an mind. drei Tagen in der Woche und durchschnittlich zwölf Zeitstunden pro Woche, die sich auch auf außerunterrichtliche Aufgaben (Konferenzteilnahmen, Wandertage, Sprachenfest, Elternabend etc.) erstrecken kann. Für das Fach Russisch gelten folgende Festlegungen:

- 30 Hospitationsstunden (à 45 Min.), um Russischunterricht theoriegeleitet mit individuell festgelegten Fragestellungen zu beobachten,

- 16 Stunden mit eigener Unterrichtstätigkeit, davon sollen mindestens neun komplett eigenständig geplant und durchgeführt werden.

Über die Beobachtung, Planung und Durchführung von Russischunterricht hinaus soll insbesondere die Auswertung des erteilten Unterrichts einen gebührenden Platz einnehmen. Wie Bechtel (2015: 79) berechtigt feststellt, ,muss den Studierenden Raum gegeben werden, angeleitet über ihre Praxiserfahrungen $\mathrm{zu}$ reflektieren, Theoriebezüge herzustellen, Handlungsalternativen zu erarbeiten, eigene Stärken und Schwächen zu analysieren und Entwicklungsaufgaben zu formulieren“. Diesen Raum bieten einerseits die Vor- und Nachbereitungsgespräche mit den MentorInnen sowie die Orientierungsgespräche zur Reflexion der eigenen Stärken und Entwicklungspotentiale und andererseits die Veranstaltungen zum „Kolle- 
gialen Unterrichtscoaching " mit dem Fachberater, aber auch die universitären Begleit-/Nachbereitungsseminare sowie die Gespräche zur Auswertung der Unterrichtsbesuche.

Zu einer weiteren Anforderung des Praxissemesters gehört die Durchführung eines Lernforschungsprojekts (LFP) an der Praktikumsschule. Dieses Projekt wird durch universitäre Begleitseminare der Erziehungswissenschaften (EWI) vorbereitet und angeleitet und dann entsprechend begleitet und betreut. Auch hier bietet sich eine Zusammenarbeit mit der Fachdidaktik an, da bei der Planung und Ausgestaltung des LFP in der Perspektive die Weiterführung der Forschungsfragen und die Nutzung der erhobenen Daten für eine Masterarbeit in der Fachdidaktik von Relevanz sein können. Aus diesem Grund erscheint es durchaus sinnvoll, bereits in den Vor- und Begleitseminaren der Fachdidaktik interessante fachspezifische Fragestellungen, die in einem LFP untersucht werden könnten, aufzugreifen und die Studierenden in ihrer Themensuche bzw. Themenanbahnung zu unterstützen.

Die Fülle der vorgestellten Begleit- und Betreuungsangebote führt zur Frage nach ihrer Effizienz und Wirksamkeit und danach, wie die PraktikantInnen diese Angebote einschätzen und bewerten.

\section{Einschätzungen und Bewertungen zur Vorbereitung und Durchführung des Praxissemesters durch Lehramts- studierende im Fach Russisch}

Die Ergebnisse der Pilotstudien zum Verlauf des Praxissemesters im Wintersemester 2016/17 an der Humboldt-Universität, die zur „Klausurtagung Praxissemester" (April 2017) vorgestellt wurden, initiierten im Bereich 
Fachdidaktik Russisch die Frage, welche konkreten Positionen die Studierenden mit dem Fach Russisch einnehmen bzw. welche Meinungen sie vertreten, wenn sie

- zum Stand ihrer Vorbereitung,

- zur Qualität der Ausbildungs- und Betreuungsangebote,

- zu ihren Erwartungen in Bezug auf das Praxissemester

befragt werden und wie sich

- ihre Antworten in die Ergebnisse der universitätsweiten Erhebungen (vgl. Körbs 2018, Körbs \& Wagner 2018, Schaumburg \& Körbs 2018) einordnen lassen.

Für den Fachbereich Russisch war in Bezug auf die Vorbereitung, Durchführung und Nachbereitung des Praxissemesters der folgenden Durchgänge von besonderem Interesse,

- in welchen Bereichen sich Probleme identifizieren lassen,

- welche Impulse für eine Optimierung gegeben werden, um die Veranstaltungen des Fachbereichs effizienter zu gestalten.

Darüber hinaus sollte der erwünschte Mehrwert der erstmalig im Sommersemester 2017 durchgeführten Mentoringqualifizierung für RussischlehrerInnen und der Einsatz des Fachberaters im Vorbereitungsseminar und während des Praxissemesters in der Reflexion der PraktikantInnen sichtbar gemacht werden.

Diese Fragestellungen sowie die dankenswerterweise ermöglichte Einsicht in eine zeitgleich verlaufende Erhebung im Bereich Fachdidaktik Englisch bildeten die Grundlage für die Erstellung des eingesetzten Fragebogens. 


\subsection{Aspekte der Befragung}

Im zweiten Durchgang des Praxissemesters (Wintersemester 2017/18) absolvierten sieben Studierende ihr Praktikum im Fach Russisch. Diese Studierenden ( $\mathrm{n}=7, \mathrm{P} 1-\mathrm{P} 7)$ wurden mittels Fragebogen um persönliche Antworten vor bzw. zu Beginn (August/September 2017) und nach bzw. zu Ende ihres Praxissemesters (Februar 2018) gebeten.

Auch wenn die Fallzahl bereits vorab als sehr klein eingestuft werden muss, wird davon ausgegangen, dass sich aus den subjektiven Sichtweisen der Studierenden in Bezug auf die Vorbereitung und Durchführung des Praxissemesters im Fach Russisch in Verbindung mit den Erziehungswissenschaften konkrete Schlussfolgerungen für die nächsten Durchgänge ableiten lassen.

Die Fragebögen zu Beginn und zum Ende des Praxissemesters (PS) enthielten jeweils 20 Fragen, die nummeriert und mit den Kürzeln B (Beginn) und E (Ende) versehen wurden, sodass z.B. die erste Frage im Fragebogen zu Beginn des PS die Bezeichnung „B-F1“ erhielt. Neben geschlossenen Fragen wurden bewusst Fragen mit Freitexteingabe aufgenommen, um Raum für subjektive Meinungsäußerungen zu bieten.

Inhaltlich lassen sich die 20 Fragen der beiden Fragebögen sieben Themenkomplexen zuordnen (vgl. I-VII, Tabelle 1), die eine zielgerichtete Auswertung ermöglichen.

\begin{tabular}{|l|c|l|}
\hline Themenkomplexe & $\begin{array}{l}\text { zum Beginn } \\
\text { des PS }\end{array}$ & $\begin{array}{l}\text { zum Ende } \\
\text { des PS }\end{array}$ \\
\hline I Studium/Studienanteile vor dem PS & \multicolumn{2}{|l|}{} \\
\hline $\begin{array}{l}\text { Fächerkombination im Bachelorstu- } \\
\text { dium, Note }\end{array}$ & B-F1 & \\
\hline $\begin{array}{l}\text { Zufriedenheit mit dem BA- und Master- } \\
\text { studium }\end{array}$ & B-F3, B-F4 & \\
\hline
\end{tabular}

Anastasia Drackert und Katrin Bente Karl (Hg.), Didaktik der slawischen Sprachen

Beiträge zum 2. Arbeitskreis in Innsbruck (19.02.-20.02.2018)

(C) 2019 innsbruck university press, ISBN 978-3-903187-80-1, DOI 10.15203/3187-80-1 


\begin{tabular}{|c|c|c|}
\hline $\begin{array}{l}\text { Relevanz der Lehrveranstaltungen für } \\
\text { das PS }\end{array}$ & B-F11 & \\
\hline $\begin{array}{l}\text { Studienanteile der Erziehungswissen- } \\
\text { schaften }\end{array}$ & B-F11b, B-F11e & \\
\hline Fachdidaktik-Module (Russisch) & $\begin{array}{l}\text { B-F11a, B- } \\
\text { F11c, d }\end{array}$ & \\
\hline \multicolumn{3}{|l|}{ II Persönliche Faktoren } \\
\hline $\begin{array}{l}\text { Unterrichtserfahrung mit (Fremd-)Spra- } \\
\text { chen }\end{array}$ & B-F2 & \\
\hline Stärken/Kompetenzen & B-F6 & E-F7 \\
\hline Überdenken der Berufswahl & B-F9 & E-F5 \\
\hline Erwartungen zum Gewinn & B-F5 & E-F8 \\
\hline III Verbindung Theorie + Praxis & B-F7 & E-F9 \\
\hline Zeitpunkt des PS & B-F8 & E-F4 \\
\hline $\begin{array}{l}\text { Anwendbarkeit der vorhandenen Kom- } \\
\text { petenzen }\end{array}$ & B-10, B-F11 & E-F6 \\
\hline IV LernbegleiterInnen & B-F13 & E-F10 \\
\hline MentorIn & B-F14 & $\begin{array}{l}\text { E-F11, E- } \\
\text { F12/13 }\end{array}$ \\
\hline Fachdidaktikerin & B-F15 & E-F14 \\
\hline Fachberater & & E-F16 \\
\hline \multicolumn{3}{|l|}{ V Forschendes Lernen } \\
\hline LFP & B-F16 & E-F17/18 \\
\hline Beobachtungsauftrag & B-F17 & E-F18 \\
\hline \multicolumn{3}{|l|}{ VI Weiteres } \\
\hline Schulische Aktivitäten & B-F18 & E-F2 \\
\hline Nebenjob & & E-F3 \\
\hline Anzahl der Unterrichtsstunden & & E-F1 \\
\hline VII Wünsche für das 4. FS & B-F19 & E-F15 \\
\hline $\begin{array}{l}\text { Das Praxissemester ist für mich gelun- } \\
\text { gen, wenn ... /weil ... }\end{array}$ & B-F20 & E-F20 \\
\hline
\end{tabular}

Tab. 1 Themenkomplexe der Fragebögen zu Beginn und Ende des Praxissemesters 
Von besonderem Interesse waren z.B. die Sichtweisen der Studierenden bzw. PraktikantInnen in Bezug auf die Verbindung von Theorie und Praxis (III), ihre Einschätzung zum Beitrag einzelner Lehrveranstaltungen (I) und zur Betreuung durch die LernbegleiterInnen (MentorInnen, Fachberater, Lehrkraft der Fachdidaktik Russisch, IV). Diese Fragestellungen waren jeweils im Fragebogen zu Beginn $\left(B-F_{x}\right)$ und zu Ende $\left(E-F_{x}\right)$ des Praxissemesters vertreten.

\subsection{Einschätzungen zur Vorbereitung auf das Praxissemester und Er- wartungen an das Praxissemester}

Von den sieben Studierenden, die an der Befragung teilnahmen, studieren fünf Russisch als erstes Fach und zwei als zweites Fach. Die jeweils anderen Fächer sind Deutsch (2x), Englisch (2x), Spanisch, Sport und Theologie. Diese Häufung im Bereich der sprachlichen Fächer eröffnete vielfältige Transfermöglichkeiten im Bereich der Fremdsprachendidaktik.

Die subjektive Zufriedenheit der Studierenden $(\mathrm{n}=7)$ mit dem Studium in der Bachelor- und Masterphase wurde mittels Frage B-F3 und B-F4 erhoben (vgl. Tabelle 2).

\begin{tabular}{|c|c|c|c|}
\hline \multicolumn{4}{|c|}{$\begin{array}{l}\text { B-F3: Wie zufrieden waren Sie insgesamt mit Ihrem BA-Studium? } \\
(\mathrm{n}=7)\end{array}$} \\
\hline sehr zufrieden & $\begin{array}{c}\text { mittelmäßig zufrie- } \\
\text { den }\end{array}$ & $\begin{array}{l}\text { gar nicht zufrie- } \\
\text { den }\end{array}$ & weiß nicht \\
\hline 1 & 5 & & 1 \\
\hline \multicolumn{4}{|c|}{ B-F4: Wie zufrieden sind Sie mit Ihrem bisherigen Master-Studium? } \\
\hline sehr zufrieden & $\begin{array}{l}\text { mittelmäßig zufrie- } \\
\text { den }\end{array}$ & $\begin{array}{l}\text { gar nicht zufrie- } \\
\text { den }\end{array}$ & weiß nicht \\
\hline 3 & 3 & & 1 \\
\hline
\end{tabular}

Tab. 2 Zufriedenheit der Befragten mit dem BA- und Masterstudium 
Zur Erklärung ihrer „Unzufriedenheit“ führten die Befragten unterschiedliche Gründe an. Auffallend hierbei ist, dass Studierende mit der Muttersprache Russisch auf Probleme mit dem Studiensystem [P2, P6] und auf den „unterschiedlichen Stil bei der Wissensvermittlung“ [P5] verwiesen. Studierende mit Russisch als Fremdsprache führten als Grund „wenig Kurse auf Russisch“ [P2], „Zu wenig Sprachpraxiskurse“ und ,zu wenig Zeit, um eigene Interessensschwerpunkte näher zu verfolgen“ [P7] an. Mehr Praxisbezug im Bachelorstudium wünschten sich zwei Befragte [P3, P6]. Dieser Praxisbezug wird demgegenüber in der Masterphase positiv hervorgehoben [P2, P3, P4]. Aus ähnlichen Gründen lässt sich erklären, warum nur zwei Studierende den Zeitpunkt des Praxissemesters (B-F8) als „genau richtig“, alle anderen als ,zu spät“ einstuften.

Der Praxisbezug der universitären Ausbildung und die gewünschte „Verbindung von Theorie und Praxis“ im Praxissemester stellen Bereiche dar, die, wie Meinungsäußerungen von Studierenden und Lehrkräften zeigen, sehr von subjektiven Einstellungen geprägt sind und deshalb verdienen, genauer betrachtet zu werden, um sie dann bei passender Gelegenheit in den (Lehr-)Veranstaltungen zu thematisieren und eine wissensbasierte Meinungsbildung zu unterstützen.

Die persönliche Sicht der Studierenden auf die „Verbindung von Theorie und Praxis" wurde mit verschiedenen Fragen zu Beginn (B-F7, B-F10, BF11) und zu Ende (E-F6, E-F9) des Praxissemesters erhoben. In den offenen Antworten (B-F7, E-F9) verwendeten die Studierenden dazu folgende Schlüsselwörter:

Theoretische Ansätze [P1], fachdidaktische Kenntnisse [P4], Gelerntes [P3, P6], Methoden [P2, P3, P7] ... in authentischen Unterrichtssituationen [P1], mit ,echten SuS" [P2, P3] ...

anwenden [P1, P2, P3, P5, P6], ausprobieren [P2, P7], erproben [P1], zur Umsetzung bringen [P4] und reflektieren [P3]. 
Nach dem Praxissemester geben die Studierenden ihre Beobachtungen u.a. wie folgt wieder:

„Die Schere zwischen Theorie und Praxis geht von Lehrkraft zur Lehrkraft unterschiedlich auseinander. Die einen unterrichten fast so, wie es die Theorie besagt, die anderen - so, wie sie es können oder wollen [P2].

Aus den Einschätzungen zum möglichen Beitrag der verschiedenen Lehrveranstaltungen in Bezug auf die Vorbereitung des Praxissemesters (BF11) ist erkennbar, dass die Studierenden dem Vorbereitungsseminar eine große Relevanz zuschreiben (vgl. Tabelle 3). Daraus folgt aber auch, dass in den anderen Veranstaltungen noch Potential besteht, den Studierenden die Bedeutsamkeit der Inhalte für die spätere Praxis aufzuzeigen bzw. die Inhalte entsprechend aufzubereiten. 


\begin{tabular}{|c|c|c|c|c|}
\hline \multicolumn{5}{|c|}{$\begin{array}{l}\text { B-F11: Wie schätzen Sie aus heutiger Sicht den Beitrag der verschiedenen } \\
\text { Lehrveranstaltungen zur Vorbereitung auf das PS ein? } \\
(\mathrm{n}=7)\end{array}$} \\
\hline Lehrveranstaltungen & $\begin{array}{c}\text { eher } \\
\text { bedeutsam }\end{array}$ & $\begin{array}{c}\text { bedingt } \\
\text { bedeutsam }\end{array}$ & $\begin{array}{c}\text { nicht } \\
\text { bedeutsam }\end{array}$ & $\begin{array}{l}\text { weiß } \\
\text { nicht }\end{array}$ \\
\hline $\begin{array}{l}\text { a) Fachdidaktik-Modul } \\
\text { im BA }\end{array}$ & 3 & 3 & & $(1)^{*}$ \\
\hline $\begin{array}{l}\text { b) Studienanteile EWI } \\
\text { im BA }\end{array}$ & 1 & 4 & 1 & $1(1)$ \\
\hline $\begin{array}{l}\text { c) Fachdidaktik-Modul } \\
5 \text { im Master- } \\
\text { Vorbereitungssemi- } \\
\text { nar }\end{array}$ & 7 & & & \\
\hline $\begin{array}{l}\text { d) Fachdidaktik-Modul } \\
6 \text { im Master- } \\
\text { Seminar Heterogeni- } \\
\text { tät }\end{array}$ & 2 & 5 & & \\
\hline $\begin{array}{l}\text { Seminar Problemfel- } \\
\text { der der Praxis }\end{array}$ & 3 & 3 & & (1) \\
\hline $\begin{array}{l}\text { e) Studienanteile EWI } \\
\text { im Master }\end{array}$ & 2 & 4 & & 1 \\
\hline
\end{tabular}

Tab. 3 Sicht der Befragten auf den Beitrag einzelner Lehrveranstaltungen

In Bezug auf ihre Vermutungen bzw. Erfahrungen, in welchem Maße sie an der Universität erworbenes Wissen im Praxissemester anwenden können (B-F10) bzw. anwenden konnten (E-F6), fallen die Bewertungen ähnlich aus. Jeweils drei Studierende entscheiden sich für ,,in hohem Maße“, die anderen vier für ,gelegentlich“. Allerdings blieb die Meinung hierzu 
nur bei drei Studierenden [P1, P3, P6] konstant; bei zwei Studierenden fielen die Vermutungen optimistischer aus als dann die Erfahrungen [P2, P4]. Die beiden anderen Studierenden [P5, P7] veränderten ihre Einschätzungen in die positive Richtung.

Die Antworten der Studierenden in Bezug auf den erwarteten persönlichen Gewinn (B-F5) spiegeln wider, dass sich die Studierenden vom Praxissemester Einblicke in das schulische Handlungsfeld erhoffen. Sie schätzen das Praxissemester als „,Realitätscheck“ [P3], Einblick in die Alltagspraxis einer Lehrkraft [P4, P3], in die reale Schulsituation [P5, P6] ein und wollen Schüleralltag aus einer anderen Sicht [P7] erleben.

Die individuellen Ergänzungen des Satzes „Das Praxissemester ist für mich persönlich gelungen, wenn ..." (B-F20) verdeutlichen die Erwartungen und Wünsche der angehenden LehrerInnen im Praxissemester,

- $\quad$ eine Bestätigung ihrer Berufswahl zu erhalten:

... ich nach den ersten (sowohl positiven als auch negativen) Erfahrungen im Unterrichten in meinem Wunsch bestärkt werde, trotzdem noch Lehrerin werden zu wollen und den Beginn des Referendariats kaum erwarten kann [P3].

... am Ende des Praktikums das Gefühl habe, in diesem Beruf bleiben zu wollen [P6]. ... mein Wunsch Lehrerin zu werden, nicht vergeht [P5].

- den Anforderungen gewachsen zu sein:

... das Gefühl habe, dem Lehrerberuf (u. zunächst natürlich erst einmal d. bevorstehendem Referendariat) gewachsen zu sein [P4].

- ,gut“ zu unterrichten:

... ich mit Sicherheit sagen kann, dass ich selbstständig eine „,gute“ Unterrichtsreihe in allen Klassenstufen planen und durchführen kann [P1]. 
... ich gute Stunden abgehalten habe und den Zugang zu meinen Schülern gefunden habe [P2].

... ich mit dem Gefühl kompetent und souverän auftreten zu können in eine Klasse gehen kann [P7].

\subsection{Einschätzungen zur Arbeit im Praxissemester}

Russischunterricht wird in Berlin an Gymnasien und Integrierten Sekundarschulen (ISS) angeboten. Entsprechend verteilen sich die Praktikumsplätze: Zwei Studierende absolvierten ihr Praxissemester an einem Gymnasium, die fünf anderen an einer ISS; zwei dieser ISS-Praktikumsschulen haben keine Oberstufe. Vier Praktikantinnen [P2, P4, P5, P7] konnten bereits vor Beginn des Praxissemesters auf eigene Unterrichtserfahrungen im Umfang von 6-18 Monaten verweisen.

Wie unter 1.1. ausgeführt, sind die MentorInnen während des Praktikums die wichtigsten Bezugspersonen für die PraktikantInnen. Bedingt durch die Schulzuweisungen wurden nur drei der sieben PraktikantInnen von MentorInnen mit einer bereits abgeschlossenen Mentoringqualifizierung betreut. Drei weitere MentorInnen absolvierten diese Qualifizierungsmaßnahme erst im Sommersemester 2018.

Wie zu vermuten war, schreiben die PraktikantInnen der Betreuung durch die MentorIn eine große Bedeutung zu (vgl. Tabelle 4). Der geringer ausfallende Stellenwert für die HU-Lehrkräfte und den Fachberater lässt sich möglicherweise durch die geringeren Kontaktzeiten erklären. 


\begin{tabular}{|c|c|c|c|c|}
\hline \multicolumn{5}{|c|}{$\begin{array}{l}\text { B-F13: Welchen Stellenwert messen Sie aus heutiger Sicht der Betreuung } \\
\text { durch die verschiedenen beteiligten Personen im Praxissemester bei? } \\
(\mathrm{n}=7)\end{array}$} \\
\hline $\begin{array}{ll}\text { Die } & \text { Betreuung } \\
\text { durch } & \end{array}$ & $\begin{array}{c}\text { eher } \\
\text { bedeutsam }\end{array}$ & $\begin{array}{l}\text { bedingt } \\
\text { bedeutsam }\end{array}$ & $\begin{array}{c}\text { nicht } \\
\text { bedeutsam }\end{array}$ & weiß nicht \\
\hline MentorIn & 7 & & & \\
\hline $\begin{array}{l}\text { Lehrkräfte der } \\
\text { HU }\end{array}$ & 4 & 3 & & \\
\hline Fachberater & 4 & 3 & & \\
\hline \multicolumn{5}{|c|}{$\begin{array}{l}\text { E-F10: Welchen Stellenwert messen Sie rückblickend der Betreuung durch } \\
\text { die verschiedenen beteiligten Personen im Praxissemester bei? }\end{array}$} \\
\hline $\begin{array}{ll}\text { Die } & \text { Betreuung } \\
\text { durch } & \end{array}$ & $\begin{array}{c}\text { eher } \\
\text { bedeutsam }\end{array}$ & $\begin{array}{l}\text { bedingt } \\
\text { bedeutsam }\end{array}$ & $\begin{array}{c}\text { nicht } \\
\text { bedeutsam }\end{array}$ & weiß nicht \\
\hline MentorIn & 6 & 1 & & \\
\hline $\begin{array}{l}\text { Lehrkräfte der } \\
\text { HU }\end{array}$ & 5 & 2 & & \\
\hline Fachberater & 2 & 4 & 1 & \\
\hline
\end{tabular}

Tab. 4 Stellenwert der Betreuung durch verschiedene LernbegleiterInnen für die Befragten

Die PraktikantInnen haben - wie ihre Einschätzungen (B-F14, E-F11) zeigen - hohe Erwartungen an den Austausch mit ihren MentorInnen, insbesondere zu Fragen des Lehrerberufs allgemein, aber auch zum Russischunterricht im Besonderen (vgl. Tabelle 5). $\mathrm{Zu}$ den anderen Aspekten verteilen sich die Erwartungen fast gleichmäßig zwischen ,eher hoch“ und „eher niedrig“. Möglicherweise ist die Sensibilisierung für die damit verbundenen Inhalte noch nicht ausreichend ausgebildet bzw. erscheinen sie den PraktikantInnen zum gegenwärtigen Zeitpunkt als nicht so relevant. 


\begin{tabular}{|l|c|c|c|c|}
\hline \multirow{2}{*}{$\begin{array}{l}\text { B-F14: Wie hoch sind Ihre Erwartungen an } \\
\text { E-F11: Wie schätzen Sie .. } \\
\text { _. die Qualität des Austausches mit Ihrer } \\
\begin{array}{l}\text { MentorIn an der Praxisschule unter } \\
\text { folgenden Gesichtspunkten ein: }\end{array}\end{array}$} & $\begin{array}{c}\text { eher } \\
\text { hoch }\end{array}$ & $\begin{array}{c}\text { eher } \\
\text { niedrig }\end{array}$ & $\begin{array}{c}\text { eher } \\
\text { hoch }\end{array}$ & $\begin{array}{c}\text { eher } \\
\text { niedrig }\end{array}$ \\
\hline Austausch zum Lehrberuf allgemein & 7 & - & 6 & 1 \\
\hline $\begin{array}{l}\text { Austausch speziell über das Unterrichten von } \\
\text { Russisch als Fremdsprache }\end{array}$ & 7 & - & 6 & 1 \\
\hline $\begin{array}{l}\text { Austausch speziell über das Unterrichten in } \\
\text { heterogenen Gruppen }\end{array}$ & 3 & 4 & 3 & 4 \\
\hline $\begin{array}{l}\text { Austausch zur gemeinsamen } \\
\text { Weiterentwicklung von Unterricht (z.B. in } \\
\text { den Klassen der MentorIn) }\end{array}$ & 4 & 3 & 4 & 3 \\
\hline $\begin{array}{l}\text { Austausch zu Diskussionen bezogen auf } \\
\text { Ihren Fachgegenstand (Russische Sprache, } \\
\text { Literatur und Kultur) }\end{array}$ & 4 & 3 & 3 & 4 \\
\hline $\begin{array}{l}\text { Austausch zu fremdsprachendidaktischen } \\
\text { Konzepten oder Fragestellungen }\end{array}$ & 3 & 4 & 5 & 2 \\
\hline $\begin{array}{l}\text { Austausch mit dem Ziel der kollegialen } \\
\text { Vernetzung }\end{array}$ & 4 & 3 & 5 & 2 \\
\hline
\end{tabular}

Tab. 5 Erwartungen der Befragten an den Austausch mit den MentorInnen

Während des Praxissemesters bereiten die PraktikantInnen in den Begleitseminaren der Erziehungswissenschaften ein Lernforschungsprojekt (LFP) vor und führen es während des Praxissemesters durch. Die Einstellungen dazu wurden vor (B-F16) und nach (E-F17) dem Praxissemester untersucht. Die Gründe für die nachweislich geringe Akzeptanz (vgl. Tabelle 6) zu Beginn des Praxissemesters liegen vermutlich darin, dass die PraktikantInnen sich (noch) nicht mit diesen Anforderungen identifizieren und den (Mehr-)Wert nicht erschließen. Die Akzeptanz verändert sich bei den PraktikantInnen, denen es gelang, das LFP mit ihrer zukünftigen Masterarbeit zu verbinden. Dieses Potential gilt es in den Erziehungswissenschaften und der Fachdidaktik verstärkt zu nutzen. 


\begin{tabular}{|c|c|c|c|}
\hline $\begin{array}{l}\text { B-F16: Bitte ge } \\
\text { im Rahmen des } \\
\text { halten. }\end{array}$ & $\begin{array}{l}\text { Sie an, für wie s } \\
\text { xissemesters für } \\
(\mathrm{n}=\end{array}$ & $\begin{array}{l}\text { voll Sie aus he } \\
\text { e professionel }\end{array}$ & klung \\
\hline sehr sinnvoll & bedingt sinnvoll & nicht sinnvoll & weiß nicht \\
\hline- & 1 & 4 & 2 \\
\hline $\begin{array}{l}\text { E-F17: Bitte ge } \\
\text { im Rahmen des } \\
\text { halten. }\end{array}$ & $\begin{array}{l}\text { ie an, für wie } \\
\text { issemesters fü }\end{array}$ & $\begin{array}{l}\text { pie aus he } \\
\text { professionell }\end{array}$ & $\begin{array}{l}\text { cht das LF } \\
\text { cklung }\end{array}$ \\
\hline sehr sinnvoll & bedingt sinnvoll & nicht sinnvoll & weiß nicht \\
\hline 1 & 3 & 3 & - \\
\hline
\end{tabular}

Tab. 6 Akzeptanz des LFP durch die Befragten

Für die Hospitationen im Russischunterricht wählen die PraktikantInnen einen spezifischen, für den Russischunterricht relevanten Beobachtungsschwerpunkt. Auch die Einstellungen dazu wurden vor (B-F17) und nach (E-F19) dem Praxissemester erfragt. Die Ergebnisse (vgl. Tabelle 7) verdeutlichen, dass die Akzeptanz hier offensichtlich etwas größer ist.

B-F17: Bitte geben Sie an, für wie sinnvoll Sie aus heutiger Sicht das Beobachtungsprojekt im Rahmen des fremdsprachendidaktischen Anteils des Praxissemesters für Ihre eigene professionelle Entwicklung halten. $(\mathrm{n}=7)$

\begin{tabular}{|c|c|c|c|}
\hline sehr sinnvoll & bedingt sinnvoll & nicht sinnvoll & weiß nicht \\
\hline 2 & 4 & - & 1 \\
\hline
\end{tabular}

E-F19: Bitte geben Sie an, für wie sinnvoll Sie rückblickend das Beobachtungsprojekt im Rahmen des fremdsprachendidaktischen Anteils des PS für Ihre eigene professionelle Entwicklung halten.

\begin{tabular}{|c|c|c|c|}
\hline sehr sinnvoll & bedingt sinnvoll & nicht sinnvoll & weiß nicht \\
\hline 2 & 5 & & \\
\hline
\end{tabular}

Tab. 7 Akzeptanz des Beobachtungsprojekts durch die Befragten 
Aber auch in diesem Bereich kommt es in der Vorbereitung und Durchführung des Praxissemesters darauf an, das Anliegen dieser Anforderungen und den erhofften Nutzen, in diesem Falle des Beobachtens, Reflektierens und Schlussfolgerns für die weitere Professionalisierung transparent und offensiv zu vertreten.

In der abschließenden Frage [E-F20] schätzen die PraktikantInnen ihr Praxissemester übereinstimmend als gelungen ein, da sie

- sich in ihrer Berufswahl bestätigt sehen [P1, P2]

- Praxis erlebt und erfahren [P2, P4, P5, P6] und einen realen und ungeschönten Einblick in den Berliner Schulalltag bekommen haben [P7].

Als persönlichen Gewinn heben sie hervor:

- stärkeres Selbstwertgefühl [P1] bzw. Selbstbewusstsein [P3],

- gewachsene Reflexionsfähigkeit [P7].

Die persönliche Sicht der befragten PraktikantInnen auf das Praxissemester ist positiv geprägt und spiegelt sich in folgenden Worten wider: Das Praxissemester hat ,,meine Erwartungen erfüllt, mir einen sehr guten Einblick in mein späteres Arbeitsfeld verschafft und mich sehr gut auf das bevorstehende Referendariat vorbereitet" [P4].

\section{Fazit}

In Vorbereitung des zweiten Durchgangs des Praxissemesters für Lehramtsstudierende an Berliner Universitäten ist es gelungen, auch für das Fach Russisch die erwünschte Verzahnung der drei Phasen der Lehr- 
kräftebildung für die weitere Professionalisierung der Studierenden auszunutzen. So wurden in enger Zusammenarbeit mit den FachseminarleiterInnen für Russisch erstmalig sowohl die Mentoringqualifizierung als auch die Fachberatung durchgeführt. Von der somit gewachsenen Zahl qualifizierter LernbegleiterInnen profitierten die Studierenden, die im Winteresemester 2017/18 ihr Praxissemester absolvierten. So wurden einige von ihnen bereits von qualifizierten MentorInnen betreut. Alle PraktikantInnen erhielten durch den Fachberater sowohl eine Einführung in ko-konstruktive Unterrichtsbesprechungen, als auch einen Einblick in den Vorbereitungsdienst.

Die Ergebnisse der Fragebogenerhebung zu Beginn und zu Ende des Praxissemesters geben anschaulich wieder, wie die Studierenden ihre Vorbereitung auf das Praxissemester und dessen Anforderungen einschätzen, welche Erwartungen sie mit dem Praxissemester verbinden und wie sie die Betreuungsangebote bewerten. Die dokumentierten Meinungen und Einschätzungen der PraktikantInnen ermöglichen den an ihrer Ausbildung beteiligten LernbegleiterInnen entsprechende Schlussfolgerungen für die weitere Optimierung der Vorbereitung und Durchführung des Praxissemesters. Als besonders wichtig erscheint hierbei die transparente und gut nachvollziehbare Darstellung aller Angebote und Anforderungen des Praxissemesters mit Blick auf den zu erwartenden (Mehr-)Wert. Das betrifft in besonderem Maße das Lernforschungsprojekt und das Beobachtungsprojekt der Fachdidaktik.

\section{Literatur}

Bechtel, M. (2015). Wandel in der Fremdsprachendidaktik gestalten. Ein Lehrer- und Lehrerinnenbildungskonzept im Rahmen der Schulbegleitforschung. In Doff, S. \& Grünewald, A. (Hrsg.), WECHSEL- 
Jahre? Wandel und Wirken in der Fremdsprachendidaktik (75-86). Trier: Wissenschaftlicher Verlag.

Fachspezifische StO/PO 2015 = Fachspezifische Studien- und Prüfungsordnung für das lehramtsbezogene Masterstudium im Fach Russisch (Schwerpunkt Gymnasium). Erstes und Zweites Fach. Überfachlicher Wahlpflichtbereich für andere Masterstudiengänge. Amtliches Mitteilungsblatt der Präsidentin (2015). Verfügbar unter: https://gremien.hu-berlin.de/de/amb/2015/59/59_2015_Russisch_Gym_PSE_30.07.15_DRUCK.pdf [28.10.2018].

$\mathrm{KMK}=$ Kultusministerkonferenz (2005). Eckpunkte für die gegenseitige Anerkennung von Bachelor- und Masterabschlüssen in Studiengängen, mit denen die Bildungsvoraussetzungen für ein Lehramt vermittelt werden. Verfügbar unter:

https://www.kmk.org/fileadmin/Dateien/veroeffentlichungen_besch luesse/2005/2005_06_02-gegenseitige-Anerkennung-BachelorMaster.pdf [28.10.2018].

Koch, C. (2015). Das Praxissemester als Reflexionsanlass: Offenlegung und Aushandeln des Selbstverständnisses der Fremdsprachendidaktik. In Doff, S. \& Grünewald, A. (Hrsg.), WECHSEL-Jahre? Wandel und Wirken in der Fremdsprachendidaktik (87-98). Trier: Wissenschaftlicher Verlag.

Körbs, C. (2018). Mentoring im Praxissemester. Ergebnisse der Evaluation der Mentoringqualifizierung. Verfügbar unter: https://pse.huberlin.de/de/forschung-und-lehre/projekte/humboldt-forschungskolleg/begleitforschung-zum-praxissemester/mentoring-im-praxissemester-ergebnisse-der.pdf [28.10.2018].

Körbs, C. \& Wagner, S. (2018). Die Fachberatung - Ergebnisse der Evaluation. Studierendenbefragung sowie Befragung der Fachseminarleitungen \& Fachdidaktiken. Verfügbar unter: https://pse.huberlin.de/de/forschung-und-lehre/projekte/humboldtforschungskolleg/begleitforschung-zum-praxissemester/2018_10_18_fachberatung_auswertung.pdf [10.11.2018].

Anastasia Drackert und Katrin Bente Karl (Hg.), Didaktik der slawischen Sprachen

Beiträge zum 2. Arbeitskreis in Innsbruck (19.02.-20.02.2018)

(C) 2019 innsbruck university press, ISBN 978-3-903187-80-1, DOI 10.15203/3187-80-1 
Kreis, A. \& Staub, F. C. (2013). Kollegiales Unterrichtscoaching. In A. Bartz, M. Dammann, S. G. Huber, T. Klieme, C. Kloft \& M. Schreiner (Hrsg.), PraxisWissen SchulLeitung (33. Aktualisierungslieferung, Teil 3, 30.32), (1-13). Köln: Wolters Kluwer.

Leitfaden Praxissemester (2018) = Freie Universität Berlin, HumboldtUniversität zu Berlin, Technische Universität Berlin, Universität der Künste Berlin (Hrsg.). Leitfaden zum Praxissemester im Berliner Lehramtsstudium. Berlin. Verfügbar unter: https://www.berlin.de/sen/bildung/fachkraefte/lehrerausbildung/studium//leitfaden_ praxissemester_05_2018_auflage3.pdf [28.10.2018].

Schaumburg, H. \& Körbs C. (2018). Studierendenbefragung zum Praxissemester. Studierbarkeit \& Mentoring (1. \& 2. Durchgang im Vergleich). Verfügbar unter: https://pse.hu-berlin.de/de/forschung-undlehre/projekte/humboldt-forschungskolleg/begleitforschung-zumpraxissemester/studierbarkeit-im-praxissemester-humboldt-tagder.pdf [28.10.2018]. 